\title{
Considering Social Determinants of Health: A Case for the Just Treatment of Vulnerable Adolescent Mental Health Inpatients
}

\author{
Melanie Goodday ${ }^{1,2}$ \\ ${ }^{1}$ Clarkson University/Icahn School of Medicine at Mount Sinai, New York City, NY, USA, ${ }^{2}$ The Garron Centre for Child and \\ Adolescent Mental Health, IWK Health Centre, Halifax, Nova Scotia, Canada
}

Correspondence: gooddam@clarkson.edu; Tel.: + 1902470 8538; Fax.: + 19024708382

Received: January 6, 2020; Accepted: February 27, 2020

\begin{abstract}
The goal of this paper is to consider the case of a vulnerable adolescent inpatient who received the standard of care, and then to propose a harm reduction approach that reflects the patient's lived-reality and better fulfills the ethics of care. For certain vulnerable adolescent mental health inpatients, the idealized treatment they receive in hospital does not align with their lived-reality upon discharge. These adolescents experience unfavorable social determinants of health (SDH). They may live in families who experience poverty, trauma, unmanaged mental illness, and lack the capacity to navigate the healthcare system. The difference between the supports these adolescents receive as inpatients and those familiar to them before admission can negatively impact their ability to get and stay well upon return to their communities. As inpatients, interdisciplinary teams can provide medication management, therapy, emotional support, proper nutrition, education, recreation, and a daily routine that promotes sleep hygiene, positive coping, and safety. These are the evidence-based ingredients that offer the best chance for recovery and management of mental illness in adolescents. If these cannot be sustained in the community, there exists an additional obligation for healthcare providers to act in the role of advocate, to ensure that the best interests of the patient are promoted through a harm reduction approach. This approach entails providing treatment options and follow-up plans that reflect and respect the patients' lived-realities even when in conflict with standard practices or hospital operating procedures. It is an approach rooted in beneficence, respect for autonomy and justice. The values of respect and compassion for the patient are promoted when healthcare providers broaden their concept of harm reduction to include bridging the gulf between idealized treatment and lived-reality outside of the hospital. Conclusion - A harm reduction approach that considers and responds to the effects of social determinants of health can better fulfill the ethics of care for vulnerable adolescent mental health inpatients.
\end{abstract}

Key Words: Adolescents • Harm Reduction - Inpatients • Mental Health • Social Determinants of Health.

\section{Introduction}

Social determinants of health (SDH) are the social and economic factors that relate to an individual's place in society, such as income, education, employment, and experiences of trauma and discrimination (1) that can affect the individual's health. Although healthcare is crucial to treating poor health, social epidemiologists argue that even more important are SDH (2). Experiences of unfavorable $\mathrm{SDH}$ are especially prevalent and associated with mental health difficulties in youth (3). Adolescents who have experienced family disruption, are adopted, or in foster care tend to be over-represented on inpatient units, and have a greater length of stay in hospital for both treatment and placement (4). They also experience barriers post-discharge in the form of unstable housing, finances, and transportation (5). The high level of support afforded to adolescent patients on mental health units can make the transition back to the community difficult (6). Research recommends integrated services that ad- 
dress SDH in youth both during inpatient treatment, and to support better engagement in outpatient follow up (5). At present, these services are often non-existent or difficult to access. To resolve this problem, I propose a harm reduction approach that can help to remediate this gap.

The aim of this paper is to set out the case of a vulnerable adolescent inpatient who received the standard of care and then propose a harm reduction approach to better fulfill the ethics of care.

\section{Case of a Vulnerable Adolescent Mental Health Inpatient ${ }^{1}$}

T.C. is a 16 y.o. male who has a diagnosis of Psychosis NOS (not otherwise specified). This is his second inpatient admission in 6 months in a region where mature minor principles apply ${ }^{2}$. T.C. has a trauma history, history of cannabis use, and regularly smokes cigarettes. During T.C.'s first inpatient admission his symptoms cleared quite quickly and he engaged fully in the recreation and school programming provided to him on the unit. He also agreed to attend counseling with his foster family. Upon discharge, he was scheduled to see his outpatient physician once a week and continue his twicedaily medication regime. Despite this, T.C. missed his first outpatient appointment, after he had to take care of his older sister's child, then continued to miss subsequent appointments. He also started avoiding home due to conflict with his foster father and began missing medication doses as a result. $\mathrm{He}$ tried to go back to school but found it difficult as his symptoms had re-emerged. He missed two of the counseling appointments and his case with the therapist was closed as a result of being categorized as a 'no show'. On his second admission, he was more ill and it took longer for his symptoms to be managed. During this current admission, his foster family is unsure whether they will accept him back

\footnotetext{
${ }^{1}$ This is a composite case and does not reflect the treatment of an actual patient. ${ }^{2}$ Mature minor principles allow children who are sufficiently mature and have met the criteria for capacity to make their own treatment decisions. This status may be determined formally by a legal process, or informally by healthcare providers (8).
}

into their home upon discharge. His options are to go to a group home, which is a residential living environment that provides assistance with daily living skills and community activities, or to the youth shelter, which is accommodation for youth experiencing homelessness that is available for a limited period of time. He is ready for discharge and has decisional capacity.

\section{Bridging the Gap}

One of the primary goals of medical care is the promotion or restoration of opportunity through the prevention or treatment of disability (7). Healthcare providers have a fiduciary duty to promote and protect patients' health and well-being. This duty becomes more complicated when the standard of care isn't effective nor able to be fully executed. The standard of care can be defined as the proper treatment for a given condition as accepted by medical experts, and widely used by healthcare providers (9). In practice, it refers to that which a minimally competent physician in the same field would do under similar circumstances (10). The standard of care for adolescent inpatients assumes that there is at least a minimally supportive social structure in place for the adolescent upon discharge. When this is not the case, the standard of care falls short of its aim. The unfavorable SDH T.C. experiences are out of the physician's control and not amenable to the standard of care. It has been proposed that physicians ought to respond to societal needs, ensure their actions reflect the communities they serve and be prepared to manage high levels of uncertainty and complexity (11). In this vein, how might a healthcare provider best ensure the promotion and protection of T.C.'s health? T.C. was unable to sustain the standard of care treatment recommendations upon discharge from his first admission. Applying the standard of care again would likely produce similar results. A different approach is warranted.

An ethically rooted alternative to the standard of care for this vulnerable adolescent population is the advocacy for, and use of a harm reduction 
approach. Harm reduction can be broadly thought of as an approach that aims to reduce the negative consequences of certain high-risk behaviours and improves health (12). I argue for an expanded view of harm reduction that includes 'certain circumstances', in addition to 'certain high-risk behaviours'. We know that circumstances, such as unfavorable $\mathrm{SDH}$, produce negative health outcomes (3), and that our behaviours are shaped by our life circumstances. In these approaches healthcare providers work with the patient to troubleshoot issues related to transition to the community in order to find solutions that may not be ideal, nor aligned with hospital policies, but that are achievable for the patient and truly reflect the principles of care. The Canadian Pediatric Society recognizes the effectiveness of harm reduction approaches, the role they can play in preventative healthcare and encourages advocating for them in hospitals, schools, and communities (13). Harm reduction is specifically endorsed for use in vulnerable populations in which certain behaviours and circumstances are already established (14). The goal of harm reduction in its broader form isn't to reduce the risky behaviour or circumstance per se, but to reduce the harm associated with the behaviour or circumstance. The healthcare provider cannot reduce the level of poverty in which a patient lives, but they can provide health interventions in ways that reduce the harm associated with this poverty. Developmentally, a harm reduction approach aligns with the tendency of adolescents to engage in experimentation and risk-taking as they become more autonomous decision-makers (13). In the next section, I aim to demonstrate that harm reduction approaches are ethically justified to promote and protect the health of vulnerable patients like T.C.

\section{What Might a Harm Reduction Approach Look Like for T.C?}

T.C. is prescribed a medication upon discharge that he will receive by injection once a month. It is covered by Pharmacare, and although not the standard of care, is a reasonable treatment option for his ill- ness. T.C. is offered the truth about the high-risk social environment at the group home and decides to stay in hospital longer than would be indicated, to engage in relationship building with his foster family as mediated by his healthcare providers. T.C. is given access to regular walks from the unit. It is known that he smokes cigarettes on hospital property during these walks. T.C. is connected with an assisted employment program instead of returning to school. A weekly standing appointment is made for him to check-in with his outpatient physician that aligns with the bus schedule and his employment programming. His foster family decides he cannot return to their home at present. He chooses to go to the youth shelter upon discharge. T.C. allows the physician to call the youth shelter the day before his appointment to remind him, and to speak with the youth workers to obtain their observations of his wellness if he starts missing appointments. If he misses a counseling appointment, his file will not be closed, the counselor will liaise with his physician to troubleshoot a new plan.

\section{Ethical Justification for a Harm Reduction Approach}

I will apply the principles of respect for autonomy, beneficence and justice to the care plan outlined above in order to illustrate its ethical foundations. Respect for autonomy is generally understood to mean that patients have the authority to make healthcare decisions for themselves (15). According to Beauchamp and Childress (16), those who make autonomous decisions act intentionally with a substantial degree of understanding and are free from internal and external controlling influences. This conceptualization of autonomy supports truth-telling, respect for privacy and confidentiality. It engenders both positive and negative obligations for healthcare providers: to disclose sufficient information to the patient, to facilitate conditions that encourage autonomous decision making, and not to subject choices and actions of patients to controlling influence (17).

Respecting T.C.'s autonomy supports an obligation for the healthcare provider to disclose the 
unpleasant truth about the high-risk environment of the group home. T.C. can only make an informed choice for himself if he has a substantial degree of understanding and appreciation for the consequences of this decision as it applies to his situation. At first glance, it may appear that living at the youth shelter would be the most harmful option. However, because the less-risky option (foster home) is no longer available to him, and T.C. is able to choose which living situation is best for him, his decision making should be supported with the aim of minimizing harm. Truth-telling in this manner may likely conflict with child welfare or hospital policies, which I will address in a later section.

There are a number of factors in a healthcare context that make autonomous decision making difficult. Among these are the vulnerability of the patient due to illness, lack of thorough understanding needed to assess the risks and benefits of treatment decisions, the power differential between patients and healthcare providers and SDH. All else being equal, we would expect vulnerable adolescents to have their autonomy on an inpatient unit further constrained by these social factors than their non-vulnerable peers. It is known that adolescents experience a loss of control, independence and privacy when they are hospitalized, which can further impair their ability to engage in decision making about their care (18).

Relational autonomy theory adds an important consideration to this model of decisional autonomy as it applies to T.C. and other vulnerable adolescents. Relational autonomy is a perspective that frames individuals as socially embedded agents who develop a sense of self through the context of social relationships; it proposes that people are inherently interdependent and interconnected. These social relationships are shaped by the intersection of $\mathrm{SDH}$ (19). It is a notion of autonomy that is sensitive to issues of social justice and is socially grounded. It takes into account the internal and external constraining effects of SDH (i.e. the psychological effects of oppression and poverty) on the autonomy of those who experience them (15). It recognizes that there is a connection between autonomy and justice and that internalizing oppressive social relations can impair autonomy (20). Specifically, the condition of autonomous choice that requires freedom from coercion may be hard to achieve for oppressed populations, in that oppression can be fundamentally restrictive to an individual's ability to make decisions (15). Therefore, respecting the autonomy of T.C. requires his healthcare providers to be attuned to the impact of his life circumstances in restricting his autonomy from the outset, and then take steps to empower him to make decisions that align with his values, goals, and preferences.

T.C. has decisional capacity, and is a voluntary patient. He has agreed to stay longer on the inpatient unit to receive additional support, but has said he will ask to be discharged if he is not able to leave the unit to smoke. He will not engage with any offers of nicotine replacement therapy. The benefits of T.C. remaining as an inpatient (relationship building with foster family, ensuring a community follow-up plan is confirmed) support respecting his autonomy by actively not restricting his liberty to leave the unit for what are effectively 'smoke breaks'.

Although smoking is objectively harmful and contravenes the policies of the hospital, the lens of harm reduction supports the healthcare providers knowingly allowing this behaviour with the aim of reducing the negative consequences of a more harmful circumstance. The circumstance, in this case, is being an inpatient, and the inherent restrictions on T.C.'s liberty and autonomy. Reducing the harm of the restriction on liberty caused by the inpatient unit is justified by the benefit achieved of T.C. remaining to receive additional support. It is expected that he will continue to smoke upon discharge and that temporary cessation of smoking during admission will not effectively improve his health. Healthcare providers should instead focus on reducing the harm to his liberty and autonomy, in order to reduce the harm that would occur if he asked to be discharged. One could use this ranking of harms to demonstrate that knowingly allowing smoking would not be justified for all patients.

It is also important not to further impair T.C.'s autonomy by rigidly prescribing a medication 
regime that he cannot sustain. A harm reduction informed approach for choosing medication would involve offering T.C. options that meet his treatment needs and that consider convenience and accessibility. T.C.'s choice to take a medication that is not the standard of care, but aligns with his ability to comply with it (financially and route of administration) should be respected. This choice is also justified by the principle of beneficence.

Beneficence is the moral obligation to benefit the patient. It must be considered in context with the principle of non-maleficence (avoiding harm), as the aims of doing good for a patient always involve the risk of producing harm (21). The obligation for the healthcare provider is to offer a net benefit to the patient. Beneficence is closely tied to the principle of respect for autonomy, as a patient with capacity can determine for themselves what constitutes a net benefit according to their values and preferences. Working with T.C. to choose an acceptably effective medication that he will adhere to, is preferable to non-adherence to the standard of care medication. This approach reduces the harm that occurs from non-adherence and is justified by beneficence.

Beneficence also supports the work of healthcare providers to engage with T.C. around building positive structure into his daily life to support his recovery. Although according to his age and social norms he should be in school, in reality, he may have been spending his days isolating at home or roaming the community using cannabis prior to the second admission to hospital. To reduce the harm of these circumstances, empowering T.C. to start a supported employment opportunity that matches his current level of functioning and is aligned with his goals, is in his best interests.

The action of the healthcare team to promote the safest living environment for T.C. is also rooted in the principle of beneficence. Actively facilitating a longer stay in hospital to give him the best shot of repairing his relationship with his foster family is harm reduction. The team is acting to increase the probability that this opportunity can be restored for him. Going to the youth shelter upon discharge is objectively non-ideal, however, if the process T.C. engaged in to come to this decision was as autonomous as possible, then the healthcare provider supporting this decision is ethically justified in supporting his choice. Implementing a communication strategy at the shelter promotes T.C.'s health in the face of circumstances that are out of his and the healthcare team's control.

One critique of harm reduction is the perception that its use implies the provider is in effect 'giving up' on the patient, or conveying to them that full recovery is neither possible nor worth pursuing. I would argue the opposite; that harm reduction demonstrates respect and compassion for the vulnerable patient because it provides the most benefit to them, by relieving the burden of illness. It uses real-time information on limitations to inform clinical decisions about risk management which in turn produces the most achievable treatment options. Healthcare providers should not act as if barriers do not exist for T.C. and his cohort, indeed that would be irresponsible. Instead, they should work with the patient to create a plan that provides the best care within these limits, while simultaneously advocating through professional avenues to break down these barriers.

The harm reduction approaches I have outlined require that vulnerable adolescents get a larger piece of the 'health care pie' according to their increased need relative to their non-vulnerable peers. Need is a material principle of justice (16). I argue from the standpoint of what distributive justice might require in order to provide these adolescents with fair equality of opportunity.

The fair equality of opportunity account of just health care is founded on the notion that healthcare goods are special because meeting health care needs has an important effect on the opportunities of individuals to choose reasonable plans of life (22). Daniels (22) argues that there is a positive societal obligation for the arrangement of services to reduce barriers that prevent fair equality of opportunity. The fair equality of opportunity account requires moral judgments to determine what types of services are needed to restore, maintain or compensate 
for the loss of normal species-typical functioning. This is relevant in that normal functioning contributes to the range of normal opportunities available to individuals (22).

I extend this argument of Daniels' to propose that under a fair equality of opportunity account, it is justified for vulnerable adolescents to receive a larger piece of the pie. The range of opportunities available to vulnerable adolescents upon discharge is diminished as compared with those of their nonvulnerable peers with the same diagnosis. When T.C. is discharged, he faces a number of barriers to the restoration of his functioning. These barriers warrant further distribution of resources (i.e. increased length of stay in hospital, additional staff time spent liaising with services in community, flexible outpatient appointments, etc.) to provide him with the range of opportunities available to his non-vulnerable peers who have social support already built into their lives upon discharge.

A resource allocation critique of this argument might question the demands these approaches place on the limited resources of the inpatient unit and on follow-up supports. This may mean decreased allocation of resources to non-vulnerable peers with the same diagnosis. A fundamental principle of justice, as defined by Aristotle, is that 'equals should be treated equally, and unequals unequally'. There would not necessarily be an equal share of resources meted out to these different patient populations, but it would be just to respect the differences between the two groups of patients by treating them equitably in this way. Those non-vulnerable peers who can sustain the treatment they receive on the inpatient unit do not need the additional support that T.C. requires.

Some of the harm reduction measures I have proposed conflict with hospital policies or procedures. Kipnis (23) argues that physicians ought to focus on what a responsible physician ought to care about and set the legal system and the impositions of the employer aside. Healthcare providers have a duty to regard the responsibility for the patient as paramount $(24,25)$, which sometimes means that what is legally or procedurally required is ethically prohibited. In T.C.'s case, it may be required by policy for a youth to be placed in a group home instead of a youth shelter. This assumes the group home is always the safer place, but when it is not, applying this standard of care will likely bring harm to him and is ethically prohibited. A provider must defy a policy when the policy and ethical treatment are in tension, and work at the systemic level to ensure that legal and institutional choices uphold professional integrity.

\section{Conclusion}

"Within the medical tradition, suffering is addressed and located in the individuals who experience it rather than in the social arrangements that may be responsible for causing the problem" (15, p. 29). The unfavorable SDH experienced by vulnerable adolescents like T.C. do impact their health, and are not able to be solved by healthcare providers in the moment. This does not absolve providers of the obligation to recognize the impact of these determinants on an individual's health and ability to engage in treatment; but in fact creates an obligation to advocate for a harm reduction approach rooted in the principles of respect for autonomy, beneficence, and justice.

Acknowledgements: This manuscript has been written as the final paper for the Clinical Ethics course of the Bioethics Certificate Program at Clarkson University, taught by Associate Professor Nada Gligorov, PhD, from the Icahn School of Medicine at Mount Sinai, New York City, NY, USA.

Conflict of Interest: The author declares that she has no conflict of interest.

\section{References}

1. Public Health Agency of Canada [homepage on the Internet]. Ottawa: Public Health Agency of Canada [updated 2019 July 25; cited 2019 Nov 04]. Social Determinants of Health and Health Inequalities. Available from: https:// www.canada.ca/en/public-health/services/health-promotion/population-health/what-determines-health.html.

2. Venkatapuram S \& Marmot M. Social Determinants of Health and Health Inequalities. In: Arras JD, Fenton E, \& 
Kukla R, editors. The Routledge Companion to Bioethics. London: Routledge; 2015. p. 301-14

3. Settipani CA, Hawke LD, Virdo G, Yorke E, Mehra K \& Henderson J. Social Determinants of Health among Youth Seeking Substance Use and Mental Health Treatment. Journal of the Canadian Academy of Child and Adolescent Psychiatry. 2018; 27(4):213-31.

4. Dickson LR, Heffron WM, \& Parker C. Children from Disrupted and Adoptive Homes on an Inpatient Unit. American Journal of Orthopsychiatry. 1990; 60(4): 594602. doi: $10.1037 /$ h0079211

5. Preyde M, Parekh S, Warne A, \& Heintzman J. School Reintegration and Perceived Needs: The Perspectives of Child and Adolescent Patients During Psychiatric Hospitalization. Child and Adolescent Social Work Journal. 2017; 34(6): 517-26. doi: 10.1007/s10560-017-0490-8

6. Gill F, Butler S, \& Pistrang N. The experience of adolescent inpatient care and the anticipated transition to the community: Young people's perspectives. Journal of Adolescence. 2006;46: 57-65. doi: 10.1016/j.adolescence.2015.10.025

7. Buchanan A, \& Brock D. Deciding for Others: The Ethics of Surrogate Decision Making (Studies in Philosophy and Health Policy). Cambridge: Cambridge University Press; 1990. doi:10.1017/CBO9781139171946.005

8. Coughlin KW. Medical decision-making in paediatrics: Infancy to adolescence. Paediatrics and Child Health. 2018; 23(2):138-46.

9. National Cancer Institute [homepage on the Internet]. Bethesda: National Institute of Health. [cited 2019 Nov 28]. Standard of Care. Available from: https://www.cancer. gov/publications/dictionaries/cancer-terms/def/standardof-care.

10. Moffett P, \& Moore G. The standard of care: Legal History and Definitions: The Bad and Good News. Western Journal of Emergency Medicine. 2011;12(1):109-12.

11. Swick H. Toward a Normative Definition of Medical Professionalism. Academic Medicine. 2000;75(6): 612-6.

12. Canadian Mental Health Association- Ontario [homepage on the Internet]. Ontario: Canadian Mental Health Association; c2019 [cited Nov. 8th 2019]. Harm Reduction. Available from: https://ontario.cmha.ca/harm-reduction/.
13. Baltzer F, Elliott A, Katzman D, Leslie K, Pinzon J, Sankaran K, \& Findlay S. Harm reduction: An approach to reducing risky health behaviours in adolescents. Paediatrics and Child Health. 2008; 13(1):53-6.

14. Toumbourou JW, Stockwell T, Neighbors C, Marlatt GA, Sturge J, Rehm J. Interventions to reduce harm associated with adolescent substance use. Lancet. 2007; 369(1):391-401.

15. Sherwin, S. A Relational Approach to Autonomy in Health Care. In: Sherwin, S and the Feminist Health Care Ethics Research Network, editors. The Politics of Women's Health: Exploring Agency and Autonomy. Philadelphia: Temple University Press; 1998. p. 19-47.

16. Beauchamp TL, \& Childress JF. Principles of Biomedical Ethics. 7th ed. New York: Oxford University Press; 2013.

17. Childress J. The Place of Autonomy in Bioethics. The Hastings Center Report. 1990;20(1):12-7.

18. Findlay S, Pinzon J, Goldberg E, Frappier J. Issues of care for hospitalized youth. Paediatrics \& Child Health. 2008;13(1):61-4. doi:10.1093/pch/13.1.61

19. Ould Brahim L. Reconsidering the 'self' in self-management of chronic illness: Lessons from relational autonomy. Nursing Inquiry. 2019; 26:(3) e12292. doi:10.1111/ $\operatorname{nin} .12292$

20. McLeod C. Self-trust and Reproductive Autonomy. Cambridge, MA: MIT Press; 2002.

21. The New Dictionary of Medical Ethics. London: BMJ Publishing; 1997. Beneficence; p.22.

22. Daniels N. Just Health Care. Cambridge and New York: Cambridge University Press; 1985.

23. Kipnis K. Medical Confidentiality. In: Rhodes R, Francis L, \& Silvers A, editors. The Blackwell Guide to Medical Ethics. Chichester: John Wiley \& Sons; 2008. p.104-27.

24. American Medical Association [homepage on the Internet]. Chicago: The Association; c1995-2019 [cited 2019 Nov 12th]. AMA Code of Medical Ethics (revised June 2001). Available from: https://www.ama-assn.org/sites/ ama-assn.org/files/corp/media-browser/principles-ofmedical-ethics.pdf.

25. Winland-Brown J, Lachman V, \& Swanson E. The new 'code of ethics for nurses with interpretive statements' (2015): Practical clinical application, part I. (ethics, law, and policy) (report). Medsurg Nursing. 2015;24(4):268. 